11-20-1996

\title{
Far-Field Scattering of a Non-Gaussian Off-Axis Axisymmetric Laser Beam by a Spherical Particle
}

James A. Lock

Cleveland State University, j.lock@csuohio.edu

Joseph T. Hodges

Follow this and additional works at: https://engagedscholarship.csuohio.edu/sciphysics_facpub

Part of the Physics Commons

How does access to this work benefit you? Let us know!

\section{Publisher's Statement}

This paper was published in Applied Optics and is made available as an electronic reprint with the permission of OSA. The paper can be found at the following URL on the OSA website: http://www.opticsinfobase.org/ao/abstract.cfm?URI=ao-35-33-6605. Systematic or multiple reproduction or distribution to multiple locations via electronic or other means is prohibited and is subject to penalties under law.

\section{Original Citation}

Lock, James A. and Joseph T. Hodges. "Far-Field Scattering of a Non-Gaussian Off-Axis Axisymmetric Laser Beam by a Spherical Particle." Applied Optics 35 (1996): 6605-6616.

\section{Repository Citation}

Lock, James A. and Hodges, Joseph T., "Far-Field Scattering of a Non-Gaussian Off-Axis Axisymmetric Laser Beam by a Spherical Particle" (1996). Physics Faculty Publications. 95.

https://engagedscholarship.csuohio.edu/sciphysics_facpub/95

This Article is brought to you for free and open access by the Physics Department at EngagedScholarship@CSU. It has been accepted for inclusion in Physics Faculty Publications by an authorized administrator of EngagedScholarship@CSU. For more information, please contact library.es@csuohio.edu. 


\title{
Far-field scattering of a non-Gaussian off-axis axisymmetric laser beam by a spherical particle
}

\author{
James A. Lock and Joseph T. Hodges
}

\begin{abstract}
Experimental laser beam profiles often deviate somewhat from the ideal Gaussian shape of the axisymmetric $\mathrm{TEM}_{00}$ laser mode. To take these deviations into account when calculating light scattering of an off-axis beam by a spherical particle, we use our phase-modeling method to approximate the beam-shape coefficients in the partial wave expansion of an experimental laser beam. We then use these beam-shape coefficients to compute the near-forward direction scattering of the off-axis beam by the particle. Our results are compared with laboratory data, and we give a physical interpretation of the various features observed in the angular scattering patterns. (c) 1996 Optical Society of America
\end{abstract}

\section{Introduction}

Among the topics that are currently of interest in classical electromagnetic scattering are the exact analytical solutions to three different extensions of the Lorenz-Mie theory, which is the theory of the scattering of a plane wave by a single spherical particle. In each of these generalizations, a different assumption required by the Lorenz-Mie scattering formalism is relaxed, i.e., (i) the plane wave is incident upon a number of neighboring or touching spherical particles or on a single spherical particle containing a number of spherical inclusions, (ii) the plane wave is incident upon a single spheroid rather than on a sphere, and (iii) the beam incident upon the spherical particle is transversely localized rather than being a plane wave. Most of the theoretical effort in the scattering of a transversely localized beam by a single spherical particle has been directed toward the scattering of a Gaussian laser beam that has been focused by a lens. ${ }^{1,2}$ This is because a usually rapidly convergent infinite series representation of the electric and magnetic fields of an axisymmetric $\mathrm{TEM}_{00}$ laser beam has long been known, ${ }^{3,4}$ and because an accurate approximation to the coefficients in the partial wave expansion of these fields has recently been

J. A. Lock is with the Department of Physics, Cleveland State University, Cleveland, Ohio 44115. J. T. Hodges is with the Chemical Science and Technology Laboratory, National Institute of Standards and Technology, Gaithersburg, Maryland 20899.

Received 12 February 1996; revised manuscript received 22 April 1996.

0003-6935/96/336605-12 $\$ 10.00 / 0$

(C) 1996 Optical Society of America developed.5-9 For the scattering of a transversely localized off-axis beam, the plane-wave scattering solution can be generalized by using the beam-shape coefficients, $A_{l m}$ and $B_{l m}$ (which occur in the partial wave expansion of the off-axis beam and scattered field). Consequently, the solution of this problem essentially consists of the determination of the beamshape coefficients for the beam of interest.

From an experimental point of view, Gaussian beam scattering is in many cases overly restrictive. The profile of an actual laser beam, though Gaussian as a zeroth order approximation, often exhibits significant deviations from the ideal Gaussian shape ${ }^{10}$ because of diffraction by dust particles on the laser mirrors, spatial nonuniformity within the laser medium, transverse mode mixing, and so on. As a result, scattering by such a beam exhibits significant deviations from the predictions of Gaussian beam scattering theory. These deviations are most apparent in the near-forward direction, where the scattered field substantially overlaps and interferes with the unscattered portion of the incident beam.

In this paper and in a previous paper, ${ }^{11}$ hereafter called paper I, we examine near-forward scattering (i.e., $|\theta| \lesssim 1^{\circ}$ ) of a non-Gaussian beam by a spherical particle. In paper I we obtained the approximate partial wave expansion of an experimental axisymmetric laser beam that struck a spherical particle head on, i.e., an on-axis scattering situation. This was accomplished by measuring the far-zone beam intensity in the absence of the particle and by modeling the shape of its surfaces of constant phase. The partial wave expansion was then used to calculate scattering by the particle, and the predicted scattered intensity was compared with experimental 


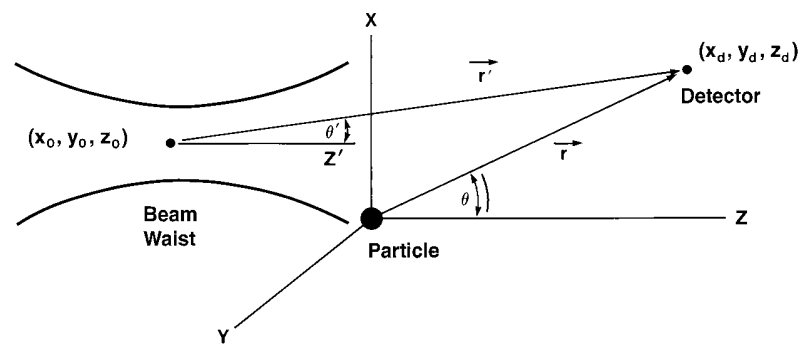

Fig. 1. Focused laser beam incident upon a spherical particle whose center is at the origin of the laboratory coordinate system. The center of the beam focal waist is at $\left(x_{0}, y_{0}, z_{0}\right)$ with respect to the particle, and the detector is located at $\left(x_{d}, y_{d}, z_{d}\right)$. The vector from the particle to the detector is $\mathbf{r}$, and the vector from the center of the focal waist to the detector is $\mathbf{r}^{\prime}$.

data. Here we extend our phase-modeling approach to an axisymmetric off-axis beam, i.e., a beam that strikes the target particle off center.

The off-axis scattering situation lacks the rotational symmetry inherent to the on-axis case. As a result, the partial wave expansion of the off-axis beam is substantially more complicated. In paper I the beam axis was coincident with an axis of symmetry of the particle, i.e., the $z$ axis in Fig. 1. As a result, the partial wave expansion of the beam was parameterized only by the partial wave number, $l$, with $1 \leq l<\infty$. For off-axis scattering, because the beam axis and the particle symmetry axis, i.e., the $z^{\prime}$ and $z$ axes, respectively, in Fig. 1, are parallel rather than coincident, the partial wave expansion of the beam is parameterized both by $l$ and by the azimuthal mode number, $m$, with $-l \leq m \leq l$.

As in paper I, we find that measuring the far-zone beam intensity and modeling the isophase surfaces of the field provide enough information to determine approximately the beam-shape coefficients in the partial wave expansion of the off-axis beam. The derivation of the beam-shape coefficients, however, is complicated by the fact that we must transform the surfaces of constant phase from one coordinate system to another that is translated from it both longitudinally and transversely. The details of this coordinate transformation also provide a physical interpretation of the beam-shape coefficients in the socalled localized approximation to off-axis Gaussian beam scattering. ${ }^{7}$

The body of this paper proceeds as follows. In Section 2 we derive our approximation to beam-shape coefficients $A_{l m}$ and $B_{l m}$ in the partial wave expansion of an experimental axisymmetric off-axis nonGaussian beam. This is accomplished in three stages. In Subsection 2.A, we obtain and simplify the partial wave expansion of an arbitrary laser beam as far as we can without having to specify any detailed information about the beam. In Subsection 2.B we model the surfaces of constant phase of the axisymmetric off-axis beam and transform them from a coordinate system whose origin is at the beam waist center to another coordinate system whose origin is at the center of the target particle. In Subsection 2.C, we connect the results of Subsections 2.A and 2.B, thus obtaining our approximation to $A_{l m}$ and $B_{l m}$. As a test of this approximation, we apply it to an off-axis Gaussian beam and obtain the localized approximation beam-shape coefficients, with minor differences. In Subsection 2.D we briefly review the formulas for the scattering of the off-axis beam by the target particle. In Section 3 we use these formulas to compute the scattering of our experimental beam, and we compare our results to laboratory data. Previous calculations of off-axis Gaussian beam scattering have been compared with laboratory data ${ }^{12,13}$ for beams up to $20 \mu \mathrm{m}$ off axis. Our data extend these measurements to beyond $110 \mu \mathrm{m}$ off axis, and a comparison of these data with the model predictions is excellent. Finally, in Section 4 we discuss the physical interpretation of our results, and we present our conclusions.

\section{Phase-Modeling Approximation to the Beam-Shape Coefficients}

\section{A. Partial Wave Expansion of an Off-Axis Focused Laser} Beam

Consider a monochromatic laser beam of wavelength $\lambda$, wave number $k=2 \pi / \lambda$, and time dependence $\exp (-i \omega t)$, which is propagating parallel to but not coincident with the $z$ axis of a laboratory coordinate system. The beam is focused by a lens, and the focal waist center is at the coordinate $\left(x_{0}, y_{0}, z_{0}\right)$ with respect to the lab coordinate system. The beam is assumed to be polarized in the $x$ direction in its focal plane. Because the radiation potentials of the beam satisfy the scalar Helmholtz equation, the partial wave expansion of the beam's electric field expressed in spherical coordinates with respect to the lab coordinate system is ${ }^{1,2}$

$$
\begin{aligned}
\mathbf{E}_{\text {beam }}(r, \theta, \phi)= & \frac{-i E_{0} \hat{u}_{r}}{(k r)^{2}} \sum_{l=1}^{\infty} \sum_{m=-l}^{l} i^{l}\left(l+\frac{1}{2}\right) \\
& \times A_{l m} \Phi_{l}(k r) P_{l}^{m}(\cos \theta) \exp (i m \phi) \\
& -\frac{i E_{0} \hat{u}_{\theta}}{k r} \sum_{l=1}^{\infty} \sum_{m=-l}^{l} \frac{i^{l}(2 l+1)}{2 l(l+1)} \\
& \times\left[A_{l m} \Phi_{l}{ }^{\prime}(k r) \tau_{l m}(\theta) \exp (i m \phi)\right. \\
& \left.-B_{l m} \Phi_{l}(k r) m \pi_{l m}(\theta) \exp (i m \phi)\right] \\
& +\frac{E_{0} \hat{u}_{\phi}}{k r} \sum_{l=1}^{\infty} \sum_{m=-l}^{l} \frac{i^{l}(2 l+1)}{2 l(l+1)} \\
& \times\left[A_{l m} \mathscr{F}_{l}{ }^{\prime}(k r) m \pi_{l m}(\theta) \exp (i m \phi)\right. \\
& \left.-B_{l m} \mathscr{F}_{l}(k r) \tau_{l m}(\theta) \exp (i m \phi)\right] .
\end{aligned}
$$

The partial wave expansion of the beam's magnetic field is similar. In Eq. (1), $\Phi_{l}(k r)$ are Riccati-Bessel functions and $P_{l}^{m}(\cos \theta)$ are associated Legendre polynomials, as was the case in paper I for an on-axis beam. Angular functions $\pi_{l m}(\theta)$ and $\tau_{l m}(\theta)$ are de- 
fined by

$$
\begin{aligned}
\pi_{l m}(\theta) & =\frac{1}{\sin \theta} P_{l}^{m}[\cos (\theta)] \\
\tau_{l m}(\theta) & =\frac{\mathrm{d}}{\mathrm{d} \theta} P_{l}^{m}[\cos (\theta)],
\end{aligned}
$$

and coefficients $A_{l m}$ and $B_{l m}$ are the beam-shape coefficients of the partial wave expansion.

Eventually, we will place a spherical particle of radius $a$ and refractive index $n$ so that its center is at the origin of the laboratory coordinate system. The waves scattered by this particle are then outgoing spherical waves centered on the origin of coordinates. In light of this, the partial wave expansion of the incident beam in Eq. (1) is appropriate because it also describes the incident beam as a collection of spherical waves centered on the origin.

As in Refs. 14 and 15, we find it convenient for computational purposes to interchange the order of the $l$ and $m$ sums in Eq. (1). We also make the following notational change:

$$
\begin{aligned}
A_{l m} & =\left\{\begin{array}{lll}
A_{l 0} & \text { for } & m=0 \\
A_{l m}{ }^{+} & \text {for } & m \geq 1 \\
A_{l m}{ }^{-} & \text {for } & m \leq-1,
\end{array}\right. \\
B_{l m} & =\left\{\begin{array}{lll}
B_{l 0} & \text { for } & m=0 \\
B_{l m}{ }^{+} & \text {for } & m \geq 1 \\
B_{l m}{ }^{-} & \text {for } & m \leq-1 .
\end{array}\right.
\end{aligned}
$$

Our major interest in the incident beam is in the far zone $(k r \gg 1)$ and in the near-forward direction $(\theta \ll$ 1 ), where the scattered wave and the unscattered portion of the incident beam substantially overlap and interfere. In this region, the Riccati-Bessel functions become

$$
\begin{aligned}
\lim _{k r \rightarrow \infty} \mathscr{g}_{l}(k r)= & \sin \left(k r-\frac{l \pi}{2}\right)=\frac{1}{2 i}\left[(-i)^{l} \exp (i k r)\right. \\
& \left.-(i)^{l} \exp (-i k r)\right],
\end{aligned}
$$

and the angular functions of Eq. (2) become

$$
\begin{aligned}
\lim _{\theta \ll 1} m \pi_{l m}(\theta)= & \frac{(l+m) !}{2(l-m) !(l+1 / 2)^{m-1}} \\
& \times\left[J_{m-1}(u)+J_{m+1}(u)\right], \\
\lim _{\theta \ll 1} \tau_{l m}(\theta)= & \frac{(l+m) !}{2(l-m) !(l+1 / 2)^{m-1}} \\
& \times\left[J_{m-1}(u)-J_{m+1}(u)\right],
\end{aligned}
$$

where $J_{m \pm 1}(u)$ are Bessel functions and

$$
u \equiv(l+1 / 2) \theta .
$$

Substituting Eqs. (3)-(6) into Eq. (1) and neglecting the radial component of the field and the incoming wave terms, i.e., those proportional to $\exp (-i k r)$, in the near-forward direction as was explained in paper
I, we obtain

$$
\begin{aligned}
\mathbf{E}_{\text {beam }}(r, \theta, \phi)= & \frac{E_{0} \hat{u}_{\theta}}{k r} \exp (i k r)\left(\frac{i}{2} \sum_{l=1}^{\infty} \frac{(l+1 / 2)^{2}}{l(l+1)} A_{l 0} J_{1}(u)\right. \\
& -\frac{i}{2} \sum_{m=1}^{\infty} \sum_{l=m}^{\infty} \frac{(l+1 / 2)^{2}}{l(l+1)} \frac{(l+m) !}{(l-m) !} \frac{1}{(l+1 / 2)^{m}} \\
& \times\left\{\left[R_{l m}{ }^{+} J_{m-1}(u)-S_{l m}{ }^{+} J_{m+1}(u)\right] \exp (i m \phi)\right. \\
& +\left[R_{l m}{ }^{-} J_{m-1}(u)-S_{l m}{ }^{-} J_{m+1}(u)\right] \\
& \times \exp (-i m \phi)\})+\frac{E_{0} \hat{u}_{\phi}}{k r} \exp (i k r) \\
& \times\left(\frac{-i}{2} \sum_{l=1}^{\infty} \frac{(l+1 / 2)^{2}}{l(l+1)} B_{l 0} J_{1}(u)+\frac{1}{2} \sum_{m=1}^{\infty} \sum_{l=m}^{\infty}\right. \\
& \times \frac{(l+1 / 2)^{2}}{l(l+1)} \frac{1}{(l+m) !} \frac{1}{(l-m) !} \frac{1 / 2)^{m}}{(l+1)} \\
& +\left\{\left[R_{l m}{ }^{+} J_{m-1}(u)+S_{l m}{ }^{+} J_{m+1}(u)\right] \exp (i m \phi)\right. \\
& -\left[R_{l m}{ }^{-} J_{m-1}(u)+S_{l m}{ }^{-} J_{m+1}(u)\right] \\
& \times \exp (-i m \phi)\} ;
\end{aligned}
$$

where $R_{l m}{ }^{ \pm}$and $S_{l m}{ }^{ \pm}$are defined by

$$
R_{l m}{ }^{ \pm} \equiv 1 / 2\left(A_{l m}{ }^{ \pm} \pm i B_{l m}{ }^{ \pm}\right), \quad S_{l m}{ }^{ \pm} \equiv 1 / 2\left(A_{l m}{ }^{ \pm} \mp i B_{l m}{ }^{ \pm}\right) .
$$

Again, the partial wave expansion of the beam's magnetic field in the far zone and in the near-forward direction is similar.

Because we expect that many partial waves are required to reconstruct the beam's electric field using Eq. (7), we may approximately replace the sum over partial waves by an integral over an effective impact parameter. Because large $m$ azimuthal modes are not expected to contribute substantially to the scattered electric field in the far zone for this off-axis situation, ${ }^{15}$ we take the lower limit of the effective impact parameter integrals to be zero rather than $m$. We also make the approximation

$$
\frac{(l+1 / 2)^{2}}{l(l+1)} \frac{(l+m) !}{(l-m) !} \frac{1}{(l+1 / 2)^{m}} \approx l^{m},
$$

which is not very accurate for low partial waves but which is quite good for the higher partial waves that we expect to provide important contributions to offaxis scattering. With these admittedly severe approximations, Eq. (7) becomes

$$
\begin{aligned}
\mathbf{E}_{\text {beam }}(r, \theta, \phi) \approx & \frac{E_{0} \hat{u}_{\theta}}{k r} \exp (i k r)\left\{\frac{i}{2} \int_{0}^{\infty} \mathrm{d} l A_{0}(l) J_{1}(l \theta)\right. \\
& -\frac{i}{2} \sum_{m=1}^{\infty} \int_{0}^{\infty} l^{m} \mathrm{~d} l\left[R_{m}^{+}(l) J_{m-1}(l \theta)\right. \\
& \left.-S_{m}{ }^{+}(l) J_{m+1}(l \theta)\right] \exp (i m \phi)
\end{aligned}
$$




$$
\begin{aligned}
& -\frac{i}{2} \sum_{m=1}^{\infty} \int_{0}^{\infty} l^{m} \mathrm{~d} l\left[R_{m}{ }^{-}(l) J_{m-1}(l \theta)\right. \\
& \left.\left.-S_{m}{ }^{-}(l) J_{m+1}(l \theta)\right] \exp (-i m \phi)\right\} \\
& +\frac{E_{0} \hat{u}_{\phi}}{k r}\left\{\frac{-i}{2} \int_{0}^{\infty} \mathrm{d} l B_{0}(l) J_{1}(l \theta)\right. \\
& +\frac{1}{2} \sum_{m=1}^{\infty} \int_{0}^{\infty} l^{m} \mathrm{~d} l\left[R_{m}{ }^{+}(l) J_{m-1}(l \theta)\right. \\
& \left.+S_{m}{ }^{+}(l) J_{m+1}(l \theta)\right] \exp (i m \phi) \\
& -\frac{1}{2} \sum_{m=1}^{\infty} \int_{0}^{\infty} l^{m} \mathrm{~d} l\left[R_{m}{ }^{-}(l) J_{m-1}(l \theta)\right. \\
& \left.\left.+S_{m}{ }^{-}(l) J_{m+1}(l \theta)\right] \exp (-i m \phi)\right\} .
\end{aligned}
$$

This is as far as we can simplify the partial wave expansion of the incident beam's electric field without knowing any particular details of the beam. In Subsection 2.B we examine the geometry of the beam, target particle, and detector and demonstrate that this geometry, along with measurement of the farzone beam intensity and modeling of the far-zone beam phase, provides enough information to approximate beam-shape coefficient functions $A_{m}(l)$ and $B_{m}(l)$ of relation (10) for an experimental beam.

\section{B. Beam-Particle-Detector Geometry}

The beam-particle-detector geometry is illustrated in Fig. 1. The particle is placed at the origin of coordinates, and the spherical coordinates with respect to this origin are $r, \theta, \phi$. The center of the beam focal waist is at the coordinate $\left(x_{0}, y_{0}, z_{0}\right)$ with respect to the particle, and the spherical coordinates with respect to the beam focal waist center are $r^{\prime}, \theta^{\prime}, \phi^{\prime}$. An element of the detector array is at the coordinate $\left(x_{d}\right.$, $\left.y_{d}, z_{d}\right)$ with respect to the particle.

As we described in paper I, each partial wave in the expansion of the incident beam is a spherical wave centered on the particle at the origin of the laboratory coordinate system. However, as seen from the far zone, the beam as a whole appears to be an outgoing spherical wave centered on beam focal waist at $\left(x_{0}\right.$, $\left.y_{0}, z_{0}\right)$. Assuming that the beam is axisymmetric about the $z^{\prime}$ axis, its far-zone electric field is a function of $r^{\prime}$ and $\theta^{\prime}$ alone, i.e.,

$$
\mathbf{E}_{\text {beam }}\left(r^{\prime}, \theta^{\prime}, \phi^{\prime}\right)=\frac{-i E_{0}}{k r^{\prime}} M\left(\theta^{\prime}\right) \exp \left(i k r^{\prime}\right) \hat{u}_{x},
$$

where $E_{0}$ is a measure of the peak field strength and the angular amplitude profile of the beam is $M\left(\theta^{\prime}\right)$.
The far-zone intensity of the beam is then

$$
I_{\text {beam }}\left(r^{\prime}, \theta^{\prime}, \phi^{\prime}\right)=\frac{E_{0}{ }^{2}}{2 \mu_{0} c} \frac{M^{2}\left(\theta^{\prime}\right)}{k^{2} r^{\prime 2}},
$$

where $\mu_{0}$ is the permeability of free space and $c$ is the speed of light.

To make contact between Eq. (11) and the partial wave expansion of the beam in approximation (10), we must convert Eq. (11) from the $r^{\prime}, \theta^{\prime}, \phi^{\prime}$ coordinates defined in Fig. 1 by

$$
\begin{aligned}
r^{\prime} & =\left[\left(x-x_{0}\right)^{2}+\left(y-y_{0}\right)^{2}+\left(z-z_{0}\right)^{2}\right]^{1 / 2}, \\
\tan \theta^{\prime} & =\frac{\left[\left(x-x_{0}\right)^{2}+\left(y-y_{0}\right)^{2}\right]^{1 / 2}}{\left(z-z_{0}\right)}, \\
\tan \phi^{\prime} & =\frac{\left(y-y_{0}\right)}{\left(x-x_{0}\right)}
\end{aligned}
$$

to the $r, \theta, \phi$ laboratory coordinates defined by

$r=\left(x^{2}+y^{2}+z^{2}\right)^{1 / 2}, \quad \tan \theta=\frac{\left(x^{2}+y^{2}\right)^{1 / 2}}{z}, \quad \tan \phi=\frac{y}{x}$.

In the near-forward direction and in the far zone where $x \gg x_{0}, y \gg y_{0}$, and $z \gg z_{0}$, we have

$$
r^{\prime} \approx r-z_{0}-\frac{x x_{0}}{z}-\frac{y y_{0}}{z}+\frac{x^{2} z_{0}}{2 z^{2}}+\frac{y^{2} z_{0}}{2 z^{2}}+\cdots .
$$

Equation (11) then becomes

$$
\begin{aligned}
\mathbf{E}_{\text {beam }}(r, \theta, \phi) \approx & \frac{-i E_{0}}{k r} M\left(x_{d}, y_{d}\right) \exp \left(-i k z_{0}\right) \hat{u}_{x} \\
& \times \exp (i k r) \exp \left[\left(i k z_{0} / 2\right) \sin ^{2} \theta\right] \\
& \times \exp \left[i k \sin \theta\left(x_{0} \cos \phi+y_{0} \sin \phi\right)\right] .
\end{aligned}
$$

Two features of approximation (16) warrant further comment. First, in the $r^{\prime}, \theta^{\prime}, \phi^{\prime}$ coordinate system, the surfaces of constant phase in Eq. (11) are represented by the $\exp \left(i k r^{\prime}\right)$ factor. In paper I where the beam was translated only longitudinally along the $z$ axis, they were represented by the $\exp [i k r+$ $\left.\left(i k z_{0} / 2\right) \sin ^{2} \theta\right]$ factor in the $r, \theta, \phi$ coordinate system. The $\theta$ dependence presented no substantial complication in determining the on-axis beam-shape coefficients. Now, because the beam is translated both longitudinally and transversely, the surfaces of constant phase are represented by the $\exp \left[i k r+\left(i k z_{0} /\right.\right.$ $\left.2) \sin ^{2} \theta+i k \sin \theta\left(x_{0} \cos \phi+y_{0} \sin \phi\right)\right]$ factor in the $r, \theta, \phi$ coordinate system. The $\phi$ dependence of these surfaces substantially complicates the determination of the off-axis beam-shape coefficients. Second, we have chosen to express $M$ as a function of $x_{d}, y_{d}$ in approximation (16) in order to emphasize that the angular amplitude profile of the beam is measured by the detector array, and that it may be expressed either in terms of $\theta^{\prime}, \phi^{\prime}$ or in terms of $\theta, \phi$ with equal 
ease by using Eqs. (13) and (14). For the geometry of the experiment described in Section 3 , we have $x_{0}=$ 0 and $x_{d}=0$ so that

$$
\phi=\phi^{\prime}= \pm \pi / 2 .
$$

The relation between $\theta^{\prime}$ and $\theta$ is then

$$
\theta^{\prime} \approx\left(\theta^{2}-2 \theta \frac{y_{0}}{z}\right)^{1 / 2}\left(1+\frac{z_{0}}{z}\right)
$$

In order to put approximation (16) into a form compatible with approximation (10) in the near-forward direction, we follow the procedure of Ref. 7 and (i) write

$$
\begin{aligned}
\hat{u}_{x}= & \cos \phi \hat{u}_{\theta}-\sin \phi \hat{u}_{\phi}=1 / 2 \exp (i \phi)\left(\hat{u}_{\theta}-i \hat{u}_{\phi}\right) \\
& +1 / 2 \exp (-i \phi)\left(\hat{u}_{\theta}+i \hat{u}_{\phi}\right)
\end{aligned}
$$

(ii) Taylor series expand the $\exp \left[i k \sin \theta\left(x_{0} \cos \phi+y_{0}\right.\right.$ $\sin \phi$ )] factor and use $\sin \theta \approx \theta$, (iii) binomial expand the resulting powers of $\left(x_{0} \cos \phi+y_{0} \sin \phi\right)$, (iv) collect together all the terms proportional to various powers of $\exp ( \pm i \phi)$, and (v) recognize the resulting infinite series as a Bessel function.

Approximation (16) then becomes

$$
\begin{aligned}
\mathbf{E}_{\text {beam }}(r, \theta, \phi)= & \frac{-i E_{0}}{k r} \exp (i k r) M\left(x_{d}, y_{d}\right) \exp \left(-i k z_{0}\right) \\
& \times \exp \left[\left(i k z_{0} / 2\right) \theta^{2}\right] \hat{u}_{\theta}\left\{-i \cos \phi_{0} J_{1}(Q)\right. \\
& +\frac{1}{2} \sum_{m=1}^{\infty}(-i)^{m-1} \exp \left[-i(m-1) \phi_{0}\right] \\
& \times \exp (i m \phi)\left[J_{m-1}(Q)-\exp \left(-2 i \phi_{0}\right)\right. \\
& \left.\times J_{m+1}(Q)\right]+\frac{1}{2} \sum_{m=1}^{\infty}(-i)^{m-1} \exp \left[i(m-1) \phi_{0}\right] \\
& \times \exp (-i m \phi)\left[J_{m-1}(Q)-\exp \left(2 i \phi_{0}\right)\right. \\
& \left.\left.\times J_{m+1}(Q)\right]\right\}-\frac{i E_{0}}{k r} \exp (i k r) \\
& \times M\left(x_{d}, y_{d}\right) \exp \left(-i k z_{0}\right) \exp \left[\left(i k z_{0} / 2\right) \theta^{2}\right] \hat{u}_{\phi} \\
& \times\left\{i \sin \phi_{0} J_{1}(Q)+\frac{i}{2} \sum_{m=1}^{\infty} \exp \left[-i(m-1) \phi_{0}\right]\right. \\
& \times \exp (i m \phi)\left[J_{\mathrm{m}-1}(\mathrm{Q})+\exp \left(-2 i \phi_{0}\right)\right. \\
& \left.\times J_{m+1}(Q)\right]-\frac{i}{2} \sum_{m=1}^{\infty} \exp \left[+i(m-1) \phi_{0}\right] \\
& \times \exp (-i m \phi)\left[J_{m-1}(Q)+\exp \left(2 i \phi_{0}\right)\right. \\
& \left.\left.\times J_{m+1}(Q)\right]\right\},
\end{aligned}
$$

where

$$
\exp \left( \pm i \phi_{0}\right)=\frac{x_{0} \pm i y_{0}}{\left(x_{0}^{2}+y_{0}^{2}\right)^{1 / 2}}
$$

$$
Q \equiv k \theta\left(x_{0}{ }^{2}+y_{0}^{2}\right)^{1 / 2} .
$$

The complexity of Eq. (20) is due solely to expressing in terms of $r, \theta, \phi$ the surfaces of constant phase that were so simply expressed in terms of $r^{\prime}, \theta^{\prime}, \phi^{\prime}$.

C. Evaluation of the Beam-Shape Coefficients

Approximation (10) and Eq. (20) each express the incident beam as a collection of outgoing spherical waves centered on the origin of the laboratory coordinate system. Consequently, we can equate the various powers of $\exp ( \pm i \phi)$ in both the $\hat{u}_{\theta}$ and $\hat{u}_{\phi}$ directions in these two relations. Furthermore, we recognize that each integral term in approximation (10) is a Hankel transform. ${ }^{16}$ Thus, by taking the inverse Hankel transforms of corresponding terms in approximation (10) and Eq. (20), we obtain

$$
\begin{aligned}
A_{0}(l)= & 2 i l \cos \phi_{0} \exp \left(-i k z_{0}\right) H_{1}(l), \\
A_{m}{ }^{ \pm}(l)= & \exp \left(-i k z_{0}\right)\left[\frac{-i \exp \left(\mp i \phi_{0}\right)}{l}\right]^{m-1} \\
& \times\left[H_{m-1}(l)+\exp \left(\mp 2 i \phi_{0}\right) H_{m+1}(l)\right], \\
B_{0}(l)= & 2 i l \sin \phi_{0} \exp \left(-i k z_{0}\right) H_{1}(l), \\
B_{m}{ }^{ \pm}(l)= & \exp \left(-i k z_{0}\right)\left(\frac{ \pm 1}{i}\right)\left[\frac{-i \exp \left(\mp i \phi_{0}\right)}{l}\right]^{m-1} \\
& \times\left[H_{m-1}(l)-\exp \left(\mp 2 i \phi_{0}\right) H_{m+1}(l)\right],
\end{aligned}
$$

where

$H_{p}(l) \equiv \int_{0}^{\infty} \theta \mathrm{d} \theta M\left(x_{d}, y_{d}\right) \exp \left(i k z_{0} \theta^{2} / 2\right) J_{p}(Q) J_{p}(l \theta)$.

Equations (23) and (24) are the main result of our study. They are our approximation to the beamshape coefficients of an off-axis axisymmetric laser beam with the far-zone intensity profile given by Eq. (12).

To test the accuracy of Eqs. (23) and (24), we apply them to an off-axis Gaussian laser beam. In the far zone (i.e., $z \gg z_{0}$ ) in this case we have $\theta^{\prime} \approx \theta$ and

$$
M(\theta)=\frac{1}{2 s^{2}} \exp \left(-\theta^{2} / 4 s^{2}\right),
$$

where $w_{0}$ is the $1 /$ e half-width of the electric field of the beam in its focal plane, and beam confinement parameter $s$ is

$$
s=1 / k w_{0} .
$$

For this Gaussian profile, the integral in Eq. (24) can be evaluated analytically, ${ }^{17}$ giving

$$
\begin{aligned}
H_{m}(l)= & \frac{1}{2 s^{2}} \int_{0}^{\infty} \theta \mathrm{d} \theta \exp \left[\frac{-\theta^{2}}{4 s^{2}}\left(1-2 i s \frac{z_{0}}{w_{0}}\right)\right] \\
& \times J_{m}\left[k\left(x_{0}{ }^{2}+y_{0}{ }^{2}\right)^{1 / 2} \theta\right] J_{m}(l \theta)=\left(1-2 i s \frac{z_{0}}{w_{0}}\right)^{-1}
\end{aligned}
$$




$$
\begin{aligned}
& \times \exp \left[\frac{-\left(x_{0}^{2}+y_{0}^{2}\right)}{w_{0}^{2}\left(1-2 i s \frac{z_{0}}{w_{0}}\right)}\right] \\
& \times I_{m}\left(\frac{2 \operatorname{sl}\left(x_{0}^{2}+y_{0}^{2}\right)}{w_{0}^{2}} \frac{1}{\left(1-2 i s \frac{z_{0}}{w_{0}}\right)}\right),
\end{aligned}
$$

where $I_{m}$ are modified Bessel functions. Insertion of this result into Eq. (23) exactly matches the localized beam-shape coefficients for an off-axis Gaussian beam found in Ref. 15, except for the replacement of $l+1 / 2$ by $l$. Because $l_{\text {max }} \approx 340$ partial waves contribute to the scattered intensity for the experiment described in Section 3, the slight mismatch between $l$ and $l+$ $1 / 2$ is of no practical consequence in our case.

This derivation also sheds light on the off-axis localized approximation for a Gaussian beam. When Gouesbet et al. ${ }^{7}$ first derived the localized version of the beam-shape coefficients for an axisymmetric offaxis Gaussian beam, they were not able to use van de Hulst's localization principle, ${ }^{18}$ as was done for the on-axis case ${ }^{19}$; instead they used the Taylor series expansion-binomial expansion procedure, as was done here in Subsection 2.B. Physically, the reason for doing so is now apparent. They had to express outgoing spherical waves centered on the beam waist in terms of outgoing spherical waves centered on the particle. This precluded the use of van de Hulst's localization principle, because it is a stationary phase argument requiring rotational symmetry, and thus it is valid only for outgoing spherical waves centered at some point along the $z$ axis.

D. Scattering of an Off-Axis Beam by a Spherical Particle The beam-shape coefficients of Eqs. (23) and (24) appear prominently in the expression for the far-zone scattered electric field produced by the interaction of an off-axis incident beam with a spherical particle whose center is at the origin of the laboratory coordinate system. The scattered electric field in the far zone is

$$
\mathbf{E}_{\text {scattered }}(r, \theta, \phi)=\frac{i \exp (i k r)}{k r}\left[S_{2}(\theta, \phi) \hat{u}_{\theta}-S_{1}(\theta, \phi) \hat{u}_{\phi}\right]
$$

where the scattering amplitudes $S_{1}(\theta, \phi)$ and $S_{2}(\theta, \phi)$ $\operatorname{are}^{14,15}$

$$
\begin{aligned}
S_{1}(\theta, \phi)= & \sum_{l=1}^{\infty} \frac{2 l+1}{2 l(l+1)} B_{l 0} b_{l} \tau_{l 0}(\theta) \\
& +\sum_{m=1}^{\infty} \sum_{l=m}^{\infty} \frac{2 l+1}{2 l(l+1)} i a_{l} m \pi_{l m}(\theta) \\
& \times\left[-A_{l m}{ }^{+} \exp (i m \phi)+A_{l m}{ }^{-} \exp (-i m \phi)\right] \\
& +\sum_{m=1}^{\infty} \sum_{l=m}^{\infty} \frac{2 l+1}{2 l(l+1)} b_{l} \tau_{l m}(\theta)\left[B_{l m}{ }^{+} \exp (i m \phi)\right. \\
& \left.+B_{l m}{ }^{-} \exp (-i m \phi)\right]
\end{aligned}
$$

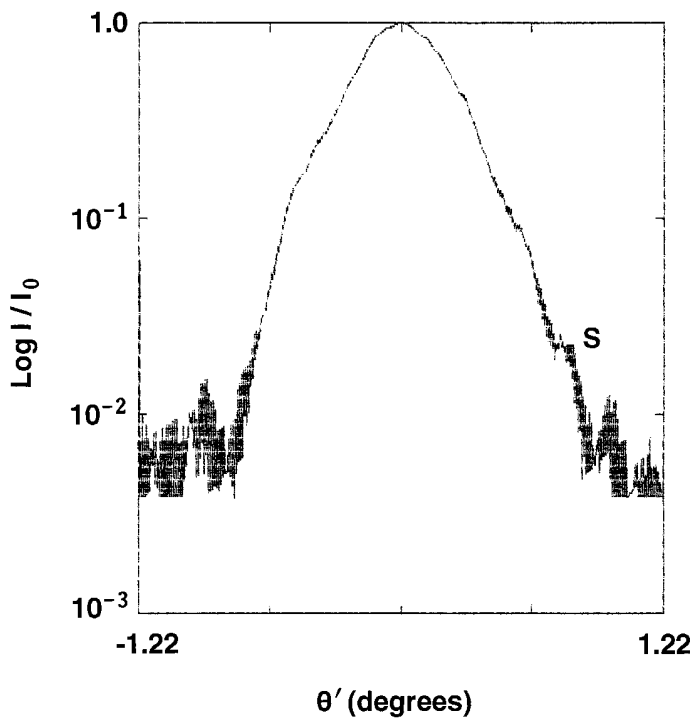

Fig. 2. Intensity as a function of angle $\theta^{\prime}$ for a focused $\mathrm{Ar}^{+}$laser beam a distance of $z_{d}{ }^{\prime}=146.2 \pm 1.5 \mathrm{~mm}$ beyond the beam focal waist. The detector is in the far zone of the beam.

$$
\begin{aligned}
S_{2}(\theta, \phi)= & \sum_{l=1}^{\infty} \frac{2 l+1}{2 l(l+1)} A_{l 0} a_{l} \tau_{l 0}(\theta) \\
& +\sum_{m=1}^{\infty} \sum_{l=m}^{\infty} \frac{2 l+1}{2 l(l+1)} a_{l} \tau_{l m}(\theta)\left[A_{l m}{ }^{+} \exp (i m \phi)\right. \\
& \left.+A_{l m}{ }^{-} \exp (-i m \phi)\right] \\
& +\sum_{m=1}^{\infty} \sum_{l=m}^{\infty} \frac{2 l+1}{2 l(l+1)} i b_{l} m \pi_{l m}(\theta) \\
& \times\left[B_{l m}{ }^{+} \exp (i m \phi)-B_{l m}{ }^{-} \exp (-i m \phi)\right],
\end{aligned}
$$

and $a_{l}$ and $b_{l}$ are the partial wave scattering amplitudes of plane-wave Lorenz-Mie theory. In the near-forward direction where overlap between the beam and scattered fields is substantial, the total field measured by the detector array is

$$
\mathbf{E}_{\text {total }}(r, \theta, \phi)=\mathbf{E}_{\text {beam }}(r, \theta, \phi)+\mathbf{E}_{\text {scattered }}(r, \theta, \phi) .
$$

\section{Experimental Validation of the Beam-Shape Coefficient Approximation}

The experimental apparatus has been described in detail both in paper I and in Ref. 10. A spatially unfiltered $\mathrm{Ar}^{+}$laser is operated at a wavelength of $\lambda$ $=0.5145 \mu \mathrm{m}$ and is focused by a single $f=100-\mathrm{mm}$ lens. At a distance of $z_{d}{ }^{\prime}=146.2 \pm 1.5 \mathrm{~mm}$ downbeam from the focal waist, the far-zone intensity profile of the beam was recorded by a CCD array having $12 \mu \mathrm{m} \times 10 \mu \mathrm{m}$ pixels, and it was normalized with respect to the peak beam intensity $I_{0}$ on the array. The normalized beam is shown in Fig. 2. Although the beam appears to be approximately Gaussian, a number of deviations from the ideal Gaussian shape are apparent. These deviations are quantified in Figs. 3(a) and 3(b), where the experimental profile is fit with a Gaussian function. For $0.25 \lesssim I / I_{0} \leq 1.0$, the beam is fit well by a Gaussian having $w_{0}=22.4$ 

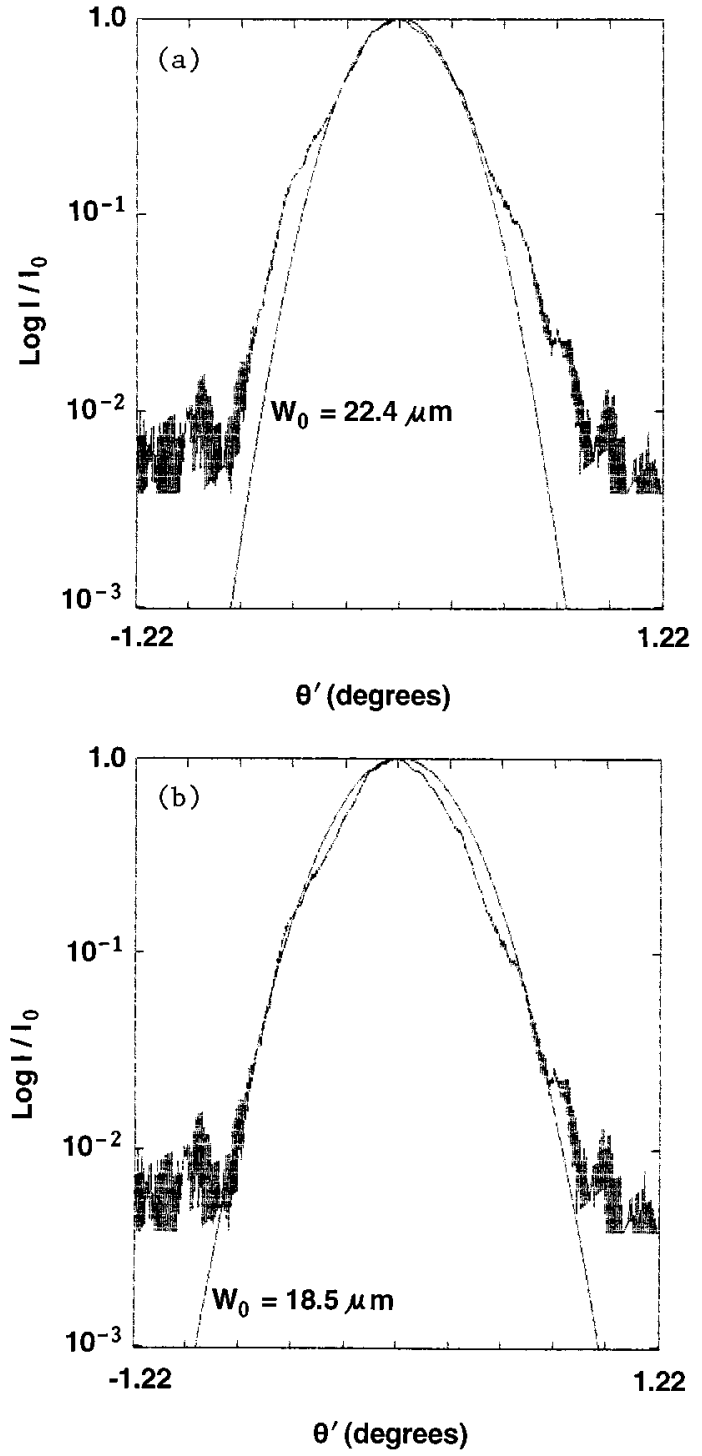

Fig. 3. Laser beam far-zone intensity profile compared with the intensity profile of a focused Gaussian beam with a focal plane electric field half-width $w_{0}$ of (a) 22.4 and (b) $18.5 \mu \mathrm{m}$.

$\mu \mathrm{m}$, whereas for $0.02 \lesssim I / I_{0} \lesssim 0.1$, it is fit well by a Gaussian having $w_{0}=18.5 \mu \mathrm{m}$. For $0.01 \lesssim I / I_{0} \lesssim$ 0.02 , the beam appears not to be axisymmetric because of the presence of the shoulder labeled $\mathrm{S}$ in Fig. 2. Thus to adapt our axisymmetric formalism of Section 2 to this beam, we consider only the intensity range $I / I_{0} \geq 0.02$.

A single droplet of dioctyl phthalate with a radius of $a=25.6 \mu \mathrm{m} \pm 0.05 \mu \mathrm{m}$ and a refractive index of $n=1.4845$ was positioned $\sim 18 \mathrm{~mm}$ downbeam from the focal waist at various positions from nominally on axis to over $110 \mu \mathrm{m}$ off axis. The beam-plusscattered light intensity was recorded on the CCD array in the near-forward direction, i.e., $|\theta| \lesssim 1^{\circ}$, and it was normalized with respect to $I_{0}$. The experimental beam-plus-scattered intensity is shown in Figs. 4(a)-4(i) for nine different particle off-axis positions with $x_{0}=0, y_{0} \neq 0$. The particle was moved nominally $\Delta y_{0} \approx 20 \mu \mathrm{m}$ between measurements, except between Figs. 4(a) and 4(b), where it was moved nominally $\Delta y_{0} \approx 40 \mu \mathrm{m}$.

Before computing the approximate beam-shape coefficients of the experimental beam of Fig. 2 and using them to compute the scattered electric field, we preprocessed the incident beam in the manner described in paper I. We determined the best-fit beam symmetry axis, averaged the intensity values of the pixels at equal distances to either side of the symmetry axis, and cut off the resulting symmetrized beam at $I / I_{0}=0.02$ in order to avoid the nonaxisymmetric feature labeled $\mathrm{S}$ in Fig. 2. We then continued the symmetrized beam to $I / I_{0}=10^{-8}$ by using the Gaussian function of Fig. 3(b) with $w_{0}=18.5 \mu \mathrm{m}$. This continuation procedure was employed in order to remove the artifacts in $H_{p}(l)$ of Eq. (24) caused by the experimental cutoff of the upper limit of the $\theta$ integral at the end of the detector array as described in paper I.

The sums over $m$ in Eqs. (29) must also be truncated at some value $m_{\max }$. This cutoff value was determined in the following way. For small off-axis distances $y_{0}$, the dominant $H_{p}(l)$ function is $H_{0}(l)$, because $J_{p}(Q) \rightarrow 0$ for $Q \ll 1$ and $p \geq 1$. For larger values of $y_{0}$, we found that many $H_{p}(l)$ functions are of roughly equal magnitude. For example, when $y_{0}$ $=100 \mu \mathrm{m}$, functions $H_{p}(l)$ were calculated for a number of values of $p$ for $1 \leq l \leq l_{\max }=343$, which is the largest partial wave that contributes more than approximately 1 part in $10^{8}$ to the plane-wave LorenzMie scattering amplitudes. It was found that as a function of $l$ for fixed $p, H_{p}(l)$ slowly oscillated, but as a function of $p$ for fixed $l$, it slowly decreased, reaching $10^{-8}$ of its $p=0$ value at $p=18$. As a result, in Eq. (29) we took $m_{\max }=19$. The relative smallness of the large $m$ terms in Eq. (29) is consistent with the convergence rate of the $m$ sums that was found for off-axis Gaussian beam scattering in Ref. 15. As a further stability test, we also computed the scattered intensity for $y_{0}=100 \mu \mathrm{m}$ and $m_{\max }=30$ and found no difference to 1 part in $10^{8}$ from the results with $m_{\max }=19$. The value $m_{\max }=19$ was used in all the far-zone intensity calculations in Figs. 4(a)-4(i). The Bessel functions in Eq. (24) were calculated by the method used in Ref. 15.

Having obtained the beam-shape coefficients of our experimental beam by using Eqs. (23) and (24), the scattered electric field was computed by using Eqs. (28) and (29). The result was added to the incident electric field of Eqs. (11), (12), and (19) to produce the total electric field of Eq. (30). The $\exp \left(-i k z_{0}\right)$ factor is common to approximation (16) and Eqs. (23) and therefore does not influence the far-zone results. The total intensity was then obtained and compared with the laboratory data. The comparison is shown in Figs. 4(a)-4(i). The only free parameters in our model are the downbeam distance, $z_{0}$, and the transverse off-axis distance, $y_{0}$. We found that as $z_{0}$ was increased, the separation between the interference maxima labeled a through e in the theoretical graphs in Figs. 4(a)-4(i) decreased. We also found that as 

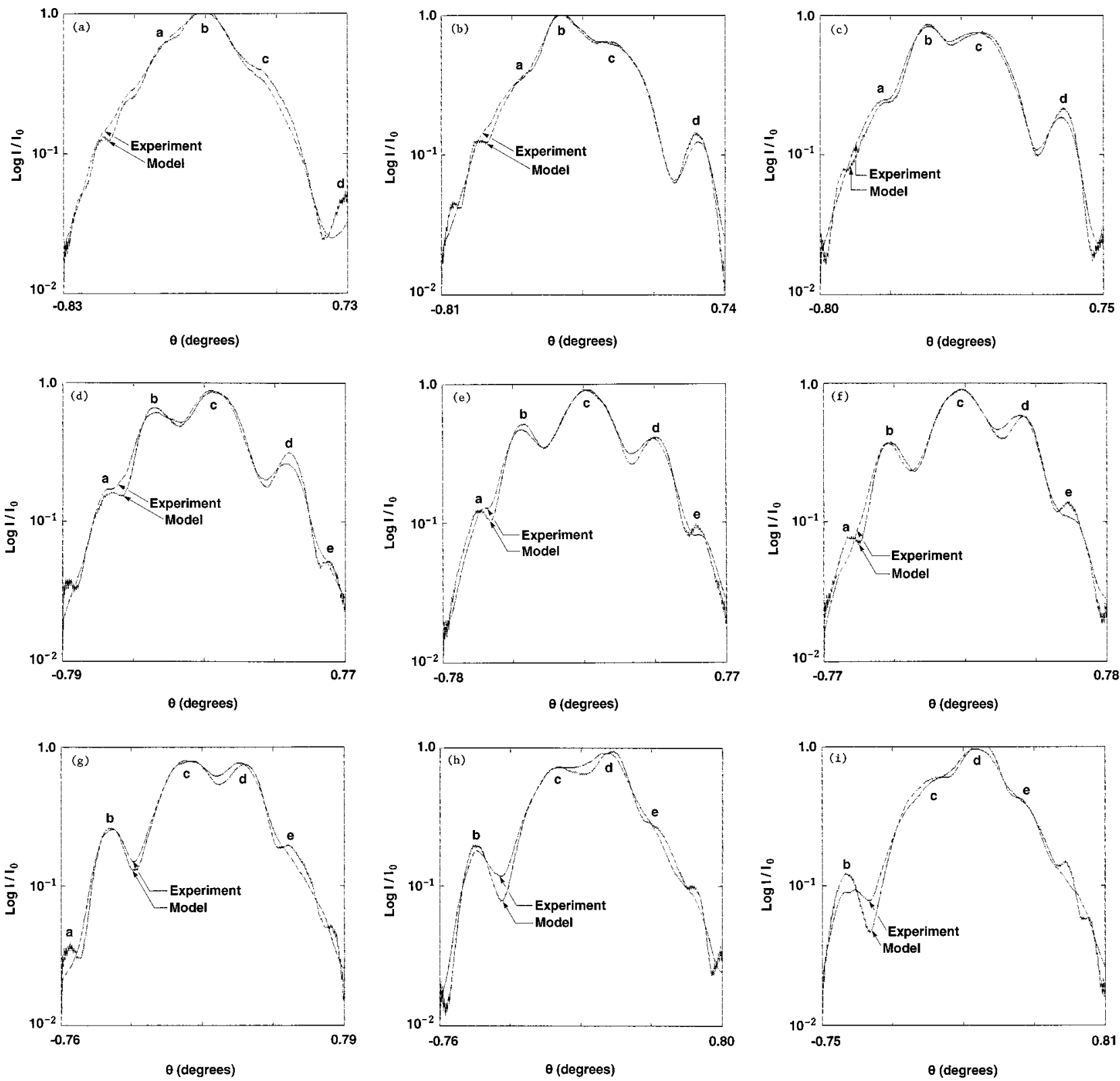

Fig. 4. Beam-plus-scattered intensity as a function of scattering angle $\theta$ for a beam waist-particle spacing of $z_{0}=-14.5 \mathrm{~mm}$, a beam off-axis position of $x_{0}=0$, and (a) $y_{0}=-114 \mu \mathrm{m}$, (b) $y_{0}=-74 \mu \mathrm{m}$, (c) $y_{0}=-52 \mu \mathrm{m}$, (d) $y_{0}=-27 \mu \mathrm{m}$, (e) $y_{0}=-7 \mu \mathrm{m}$, (f) $y_{0}=11 \mu \mathrm{m}$, (g) $y_{0}=31 \mu \mathrm{m}$, (h) $y_{0}=48 \mu \mathrm{m}$, (i) $y_{0}=66 \mu \mathrm{m}$.

$y_{0}$ was made more negative, the theoretical intensity for $\theta<0$ increased and the theoretical intensity for $\theta$ $>0$ decreased. Thus by varying $z_{0}$ and $y_{0}$, we could adjust our theoretical intensity to match the experimental intensity.

To evaluate downbeam distance $z_{0}$, we used the on-axis fitting procedure of paper I first with the nominally on-axis data of Fig. 4(e). This case, which gave a good comparison for $z_{0}=-14.5 \mathrm{~mm}$ as shown in Fig. 5, is consistent with Fig. 3(b) of paper I. Though the fit in Fig. 5 is good, the theoretical peaks labeled a and e are too low and too high, respectively, indicating that the particle was slightly off axis. The intensity for off-axis scattering was then computed and a good comparison was obtained for $z_{0}=-14.5$ $\mathrm{mm}$ and $y_{0}=-7 \mu \mathrm{m}$, as is shown in Fig. 4(e). The value of $x_{0}$ was also varied, and a good comparison was obtained for $x_{0}=0$. The comparison deteriorated as $\left|x_{0}\right|$ was increased.

At this point, fits for the other eight measured spectra of Fig. 4 were performed. Fixing the downbeam distance of the particle from the focal waist at $z_{0}=$ $-14.5 \mathrm{~mm}$, and with $x_{0}=0$, we found a good comparison with the experimental data for $y_{0}=-114 \mu \mathrm{m}$ for Fig. 4(a), $y_{0}=-74 \mu \mathrm{m}$ for Fig. 4(b), $y_{0}=-52 \mu \mathrm{m}$ for Fig. 4(c), $y_{0}=-27 \mu \mathrm{m}$ for Fig. 4(d), $y_{0}=-7 \mu \mathrm{m}$ for Fig. 4(e), $y_{0}=11 \mu \mathrm{m}$ for Fig. 4(f), $y_{0}=31 \mu \mathrm{m}$ for Fig. $4(\mathrm{~g}), y_{0}=48 \mu \mathrm{m}$ for Fig. $4(\mathrm{~h})$, and $y_{0}=66 \mu \mathrm{m}$ for Fig. 4(i). Figures 4(a)-4(i), mirroring the placement of the CCD array, are centered with respect to the 


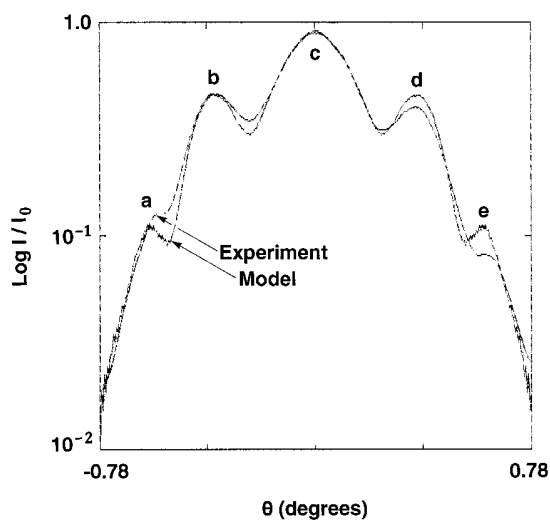

Fig. 5. Beam-plus-scattered intensity as a function of scattering angle $\theta$ for the experimental data of Fig. 4(e) and our on-axis model with $z_{0}=-14.5 \mathrm{~mm}$ and $x_{0}=y_{0}=0$.

incident beam. Thus the horizontal axis of the graphs would be symmetric with respect to $\theta^{\prime}$. The graphs here are plotted with respect to scattering angle $\theta$, however, resulting in the horizontal axis being asymmetric. The fitted transverse distances between measurements compare favorably with the nominal $20-\mu \mathrm{m}$ experimental distance. A leastsquares calculation of the fitted $\Delta y$ values to the experimental (i.e., micrometer based) $\Delta y$ values gave a slope within $1 \%$ of unity. For each of the theoretical comparisons in Figs. 4(a)-4(i), $x_{0}$ and $y_{0}$ could each be varied by $\pm 2 \mu \mathrm{m}$ and $z_{0}$ could be varied by $\pm 0.5 \mathrm{~mm}$ from the above results and still give a good comparison to the experimental data. We consider the agreement between the predictions of our model and the off-axis laboratory data to be excellent. The greatest difference between the theoretical fits and the experimental data is that the contrast between the interference maxima and minima in the data is smaller than that predicted by our model. This was also noticed in paper I, and its origin is uncertain. The occasional appearance of small amplitude highfrequency oscillations in our theoretical fits is due to a small discontinuity in $M\left(x_{d}, y_{d}\right)$ at $I / I_{0}=0.02$, inadvertently produced by the continuation procedure in the preprocessing of the beam.

A trade-off has been made in this experiment with respect to previous far-zone near-forward-direction scattering experiments that were then compared with either the Gaussian beam scattering theory ${ }^{13}$ or the diffraction theory. ${ }^{12}$ Our off-axis range of $y_{0}$ is substantially larger than that of previous experiments. With $y_{0}$ normalized with respect to the local beam half-width at the position of the particle, we have $y_{0}{ }^{\max } / w\left(z_{0}\right)=1.05$, whereas Ref. 13 had $y_{0}{ }^{\max } /$ $w\left(z_{0}\right)=0.34$ and Ref. 12 had $y_{0}{ }^{\max } / w\left(z_{0}\right)=0.47$. However, as the dynamic range of our CCD array was limited, we were confined to $I_{\text {scattered }} / I_{0} \gtrsim 2 \times 10^{-2}$, whereas previous experiments reported measurements for $I_{\text {scattered }} / I_{0} \gtrless 10^{-5}$ and $I_{\text {scattered }} / I_{0} \gtrsim 10^{-3}$. As a result, we must exercise some caution when comparing our results with those of previous experiments, because previous authors may have sacrificed some quality of the fits for $I_{\text {scattered }} / I_{0} \geqslant 0.02$ in order to obtain better fits for $I_{\text {scattered }} / I_{0} \lesssim 0.02$.

With this qualification in mind, we see that the Gaussian beam diffraction theory works well in Ref. 12 largely because the spatially filtered beam in that experiment had almost an exact Gaussian profile. In contrast, we believe that the quality of our fits for $I_{\text {scattered }} / I_{0} \geq 0.02$ is superior to that reported by Guilloteau et al., ${ }^{13}$ who employed Gaussian beam scattering theory to fit the data generated by the spatially unfiltered incident beam in that experiment. They claimed that their fit was

"always quite satisfactory in the inner zone. The amplitudes and locations of the inner lobes [our maxima b, c, and d in Figs. 4(a)-4(i)] and the modifications of the patterns with respect to the control parameter $x_{0}$ [we used $y_{0}$ ] are remarkably well predicted by the theory. Deterioration of the agreement occurs when we approach the frontier of the inner zone (where the unscattered portion of the incident beam ceases overlapping with the scattered wave) for $|\theta|>2^{\circ}$ (where $I_{\text {scattered }} / I_{0} \approx 10^{-1}$ ) as expected and previously discussed."

These expected differences are attributed by Guilloteau et al. ${ }^{13}$ to "imperfections in the Gaussian character of the beam ... because of the finiteness of the laser source." An examination of Figs. 4(a)-4(i) shows that the fit between our model and our off-axis data is somewhat superior to the fit of Ref. 13 for $I_{\text {scattered }} / I_{0} \geqslant 0.1$, and it is markedly superior for 0.02 $\lesssim I_{\text {scattered }} / I_{0}<0.1$. Thus we believe that our phase-modeling approximation of non-Gaussian beam scattering remedies the difficulties that were encountered by Guilloteau et al. ${ }^{13}$ in fitting the finer features of their off-axis scattering data for $I_{\text {scattered }} / I_{0} \lesssim 0.1$.

We did not attempt to fit our experimental data by using the Gaussian beam scattering theory. As was seen in Figs. 3(a) and 3(b), the central portion of our beam, $0.25 \leq I / I_{0} \lesssim 1.0$, is fit well by a Gaussian of one width while the outer portions of the beam, 0.02 $\lesssim I / I_{0} \lesssim 0.1$, are fit well by a Gaussian of another width. Correspondingly, it was shown in Ref. 10 that the Gaussian beam scattering theory would either fit the central portion of our experimental data well while underestimating the outer portions of the data, or it would fit the outer portions of our data well while overestimating the central portion of the data.

\section{Discussion}

In recent years, three theories have been employed by various authors to fit far-zone near-forward direction scattering in the angular region where the unscattered portion of the incident beam overlaps the scattered wave and interferes with it. These theories are (i) the diffraction theory, ${ }^{12}$ (ii) the Gaussian beam scattering theory, ${ }^{13}$ and (iii) the non-Gaussian beam scattering theory, which we employ here and in paper I. The diffraction theory is both the simplest and most approximate of the theories. The Gaussian beam scattering theory is a generalization of it, and the non-Gaussian scattering theory constitutes a fur- 
ther generalization. The generalization is related to the fact that the dominant physical processes for the scattering of a transversely localized beam in the near-forward direction by a large spherical particle, but with the particle radius smaller than the local beam radius at the position of the particle, are diffraction, reflection of near-grazing rays, and transmission of near-axial rays. ${ }^{14}$ Further, diffraction dominates for $\theta$ in the range of the first few oscillations of the diffracted field. ${ }^{20}$ For our case with $a=25.6$ $\mu \mathrm{m}$ and $\lambda=0.5145 \mu \mathrm{m}$, the first diffraction zero occurs at $\theta= \pm 0.70^{\circ}$. Diffraction is thus dominant over the entire angular interval of Figs. 4(a)-4(i), making theory (i) a good zeroth order approximation in describing the scattering. In theories (ii) and (iii), all of the other weaker scattering mechanisms such as reflection and transmission are also included. In (ii) the strengths of these mechanisms are modulated by the Gaussian profile of the beam, ${ }^{6}$ and in (iii) they are modulated by the arbitrary profile of the axisymmetric beam.

When examining the laboratory scattering data of Figs. 4(a)-4(i), we see that a prominent feature is the sequence of interference peaks labeled a through e. These peaks have the following physical interpretation. Because the first diffraction zero occurs at $\theta=$ $\pm 0.70^{\circ}$, the oscillations of the diffracted field cannot be responsible for the peaks. As described in paper I, the peaks are caused by the interference of the forward lobe of the diffracted wave, whose surfaces of constant phase have the radius of curvature $r$ at the detector plane, with the unscattered portion of the incident beam, whose surfaces of constant phase have the radius of curvature $r^{\prime}$ there. Any model for the incident beam, either Gaussian or non-Gaussian, will fit the experimental positions of the peaks accurately, as long as it includes both diffraction and the interference of the incident beam with the scattered or diffracted wave. This was seen both in Refs. 12 and 13 and in Figs. 4(a)-4(i) here.

For our experiment, the beam is sufficiently wide with respect to the target particle that the intensity of the scattered wave is more than an order of magnitude weaker than the intensity of the incident beam. This disparity is shown in Fig. $6(\mathrm{~b})$ for $y_{0}=0$. Thus the interference of the beam and the scattered wave oscillates rather closely about the incident beam profile. As a result, if one were to model inaccurately the angular dependence of the incident beam profile, i.e., $M\left(\theta^{\prime}\right)$ in Eq. (11), the interference maxima and minima would be in the right places. However, the baseline curve upon which they are superposed would be incorrect [See, e.g., the Gaussian beam model for Figs. 3(b) and 3(c) of paper I]. The fits to both the positions of the interference maxima and minima, as well as the shape of the overall intensity curve, are accurate only when the incident beam amplitude profile is modeled accurately, as we have done here in Section 2.

A second noticeable feature in Figs. 4(a)-4(i) is the variable peak-to-valley contrast of the interference structure. For $y_{0}<0$, the contrast of the oscillations for $\theta>0$ is increased while the contrast for $\theta<0$ is
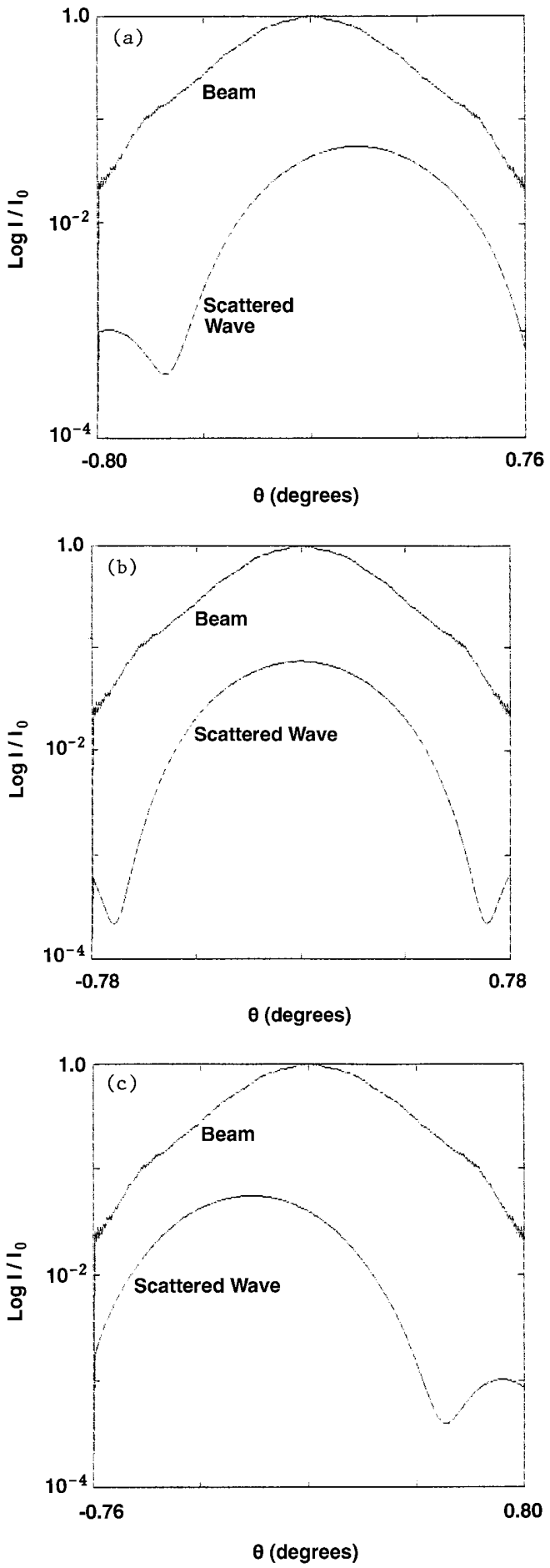

Fig. 6. Scattered intensity and beam intensity as a function of scattering angle $\theta$ for $z_{0}=-14.5 \mathrm{~mm}, x_{0}=0$, and (a) $y_{0}=-50 \mu \mathrm{m}$, (b) $y_{0}=0$, (c) $y_{0}=50 \mu \mathrm{m}$.

decreased. Similarly, for $y_{0}>0$ the contrast for $\theta<$ 0 is increased while the contrast for $\theta>0$ is decreased. As shown in Figs. 6(a) and 6(c) for $y_{0}=$ $-50 \mu \mathrm{m}$ and $y_{0}=50 \mu \mathrm{m}$, respectively, this is a consequence of the off-axis geometry of the beam and particle. For $y_{0}<0$ in Fig. 6(a) the incident beam shifts to negative $\theta$ so that the forward diffraction lobe 
is more comparable in amplitude to the $\theta>0$ portion of the incident beam, leading to a larger-contrast interference structure. The forward diffraction lobe is also less comparable in amplitude with the $\theta<0$ portion of the incident beam, leading to a smallercontrast interference structure. In Fig. 6(c) for $y_{0}>$ 0 where the incident beam shifts to positive $\theta$, the behavior of the contrast of the interference structure is reversed. Lastly, it should also be noticed that in Figs. 4(a) and 4(b) the peak value of the beam-plusscattered intensity is larger than the intensity of the beam alone in the absence of the particle. This is due to constructive interference of the diffracted wave and the incident beam at the center of the beam when $\left|y_{0}\right|$ is larger than approximately $70 \mu \mathrm{m}$.

Our final observation is that there are many approximations that one must consider when implementing a particular version of the scattering theory. These approximations are not immediately apparent when one is examining the formal exact equations of the theory. In recent years this has been seen to be the case for the Gaussian beam scattering theory. One can evaluate beam-shape coefficients $A_{l m}$ and $B_{l m}$ exactly by performing either a two-dimensional ${ }^{2}$ or a three-dimensional integral ${ }^{1}$ over the radial component of the beam's electric and magnetic fields, but only if those field components are known exactly. The exact fields for a Gaussian beam, unfortunately, are not known, although a complicated but usually rapidly convergent infinite series representation for them is available. ${ }^{3,4}$ However, upon truncating the infinite series at some point, one finds that the value of the two-dimensional integral for $A_{l m}$ and $B_{l m}$ weakly depends on the choice of the radial evaluation point, and various contributions to the threedimensional integral for $A_{l m}$ and $B_{l m}$ diverge. ${ }^{8}$

As an alternative, a localized approximation for $A_{l m}$ and $B_{l m}$ for both on-axis ${ }^{5,6}$ and off-axis ${ }^{7}$ Gaussian beam scattering was developed. The approximation is usually quite accurate, and in retrospect ${ }^{21}$ it was found that the localized approximation anticipates many of the higher-order terms in the infinite series expansion of the beam fields. From a different point of view, an arbitrary specification of a set of $A_{l m}$ and $B_{l m}$ coefficients describes a beam that is an exact solution of Maxwell's equations. ${ }^{8,21}$ Such a set of coefficients has been constructed so as to approximate accurately a Gaussian beam near its focal waist. However, the behavior of the resulting beam far from the focal waist has yet to be studied.

Similar difficulties also occur in the formal equations for non-Gaussian beam scattering. The theory can be exactly written down, as was done in Eqs. (1), (28), and (29). However, when one wishes to evaluate beam-shape coefficients $A_{l m}$ and $B_{l m}$, a number of approximations of one sort or another must be made. Without knowing the exact functional form of the radial component of the electric and magnetic fields of an experimental beam, we cannot evaluate $A_{l m}$ and $B_{l m}$ by direct integration. The localized on-axis beam-shape coefficients are generalizable for an axisymmetric beam because of their derivation by means of stationary phase arguments, but again the exact beam profile must be known. And, even if it were, the generation of the off-axis coefficients from the on-axis coefficients by means of translation theorems for vector spherical harmonics is not yet well developed. ${ }^{22}$ Furthermore, to our knowledge no theory has yet been devised to approximate $A_{l m}$ and $B_{l m}$ for a nonaxisymmetric beam either, on axis or off axis.

Again as an alternative, our approximation based on measuring the beam's far-zone intensity profile and modeling its far-zone phase behavior is given by Eqs. (23) and (24). In this paper we have shown that this approximation is physically motivated, and that it is an axisymmetric non-Gaussian beam generalization of the localized approximation. By comparison with laboratory data in Figs. 4(a)-4(i) we have shown that, at least in this case, it is also quite accurate. It is of interest to compare our model to experimental data for a more severely non-Gaussian beam, such as an axisymmetric higher-order laser mode. We anticipate that our model would produce an accurate approximation for scattering by such a beam, provided that the phase modeling of the beam fields is still described by the spherical wave fronts of Eq. (11).

We thank Gérard Gouesbet of l'Institut National des Sciences Appliquées de Rouen, France, for bringing the problem of obtaining the beam-shape coefficients of an actual laser beam to our attention.

\section{References}

1. G. Gouesbet, B. Maheu, and G. Gréhan, "Light scattering from a sphere arbitrarily located in a Gaussian beam, using a Bromwich formulation,” J. Opt. Soc. Am. A 5, 1427-1443 (1988).

2. J. P. Barton, D. R. Alexander, and S. A. Schaub, "Internal and near-surface electromagnetic fields for a spherical particle irradiated by a focused laser beam,” J. Appl. Phys. 64, 16321639 (1988).

3. L. W. Davis, "Theory of electromagnetic beams," Phys. Rev. A 19, 1177-1179 (1979).

4. J. P. Barton and D. R. Alexander, "Fifth-order corrected electromagnetic field components for a fundamental Gaussian beam,” J. Appl. Phys. 66, 2800-2802 (1989).

5. G. Gréhan, B. Maheu, and G. Gouesbet, "Scattering of laser beams by Mie scatter centers: numerical results using a localized approximation," Appl. Opt. 25, 3539-3548 (1986).

6. B. Maheu, G. Gréhan, and G. Gouesbet, "Ray localization in Gaussian beams," Opt. Commun. 70, 259-262 (1989).

7. G. Gouesbet, G. Gréhan, and B. Maheu, "Localized interpretation to compute all the coefficients $g_{n}{ }^{m}$ in the generalized Lorenz-Mie theory," J. Opt. Soc. Am. A 7, 998-1007 (1990).

8. J. A. Lock and G. Gouesbet, "Rigorous justification of the localized approximation to the beam-shape coefficients in generalized Lorenz-Mie theory. I. On-axis beams," J. Opt. Soc. Am. A 11, 2503-2515 (1994).

9. G. Gouesbet and J. A. Lock, "Rigorous justification of the localized approximation to the beam-shape coefficients in generalized Lorenz-Mie theory. II. Off-axis beams," J. Opt. Soc. Am. A 11, 2516-2525 (1994).

10. J. T. Hodges, G. Gréhan, G. Gouesbet, and C. Presser, "Forward scattering of a Gaussian beam by a nonabsorbing sphere," Appl. Opt. 34, 2120-2132 (1995).

11. J. A. Lock and J. T. Hodges, "Far-field scattering of an axisymmetric laser beam of arbitrary profile by an on-axis spherical particle,” Appl. Opt. 35, 4283-4290 (1996). 
12. J.-P. Chevaillier, J. Fabre, and P. Hamelin, "Forward scattered light intensities by a sphere located anywhere in a Gaussian beam," Appl. Opt. 25, 1222-1225 (1986).

13. F. Guilloteau, G. Gréhan, and G. Gouesbet, "Optical levitation experiments to assess the validity of the generalized LorenzMie theory," Appl. Opt. 31, 2942-2951 (1992).

14. J. A. Lock, "Contribution of high-order rainbows to the scattering of a Gaussian laser beam by a spherical particle," J. Opt. Soc. Am. A 10, 693-706 (1993).

15. J. A. Lock, "Improved Gaussian beam scattering algorithm," Appl. Opt. 34, 559-570 (1995).

16. G. Arfken, Mathematical Methods for Physicists, 3rd ed. (Academic, New York, 1985), pp. 795-797.
17. I. S. Gradshteyn and I. M. Ryzhik, Table of Integrals, Series, and Products (Academic, New York, 1965), p. 718, no. 6.633.2.

18. H. C. van de Hulst, Light Scattering by Small Particles (Dover, New York, 1981), pp. 208-209, 210-214.

19. Ref. 14, Appendix A.

20. H. M. Nussenzveig and W. J. Wiscombe, "Complex angular momentum approximation to hard-core scattering," Phys. Rev. A 43, 2093-2112 (1991).

21. G. Gouesbet, J. A. Lock, and G. Gréhan, "Partial-wave representations of laser beams for use in light-scattering calculations," Appl. Opt. 34, 2133-2143 (1995).

22. A. Doicu, Department of Fine Mechanics and Optics, Politehnica, University of Bucharest, Romania (personal communication, 1994). 